\title{
Expectancy Violations about Physical Properties of Animated Objects in Dogs
}

\author{
Christoph J. Völter (Christoph.Voelter@vetmeduni.ac.at) \\ Ludwig Huber (Ludwig.Huber@vetmeduni.ac.at)
}

\begin{abstract}
Comparative Cognition, Messerli Research Institute, University of Veterinary Medicine Vienna, Medical University of Vienna, University of Vienna, Veterinaerplatz 1, 1210 Vienna, Austria
\end{abstract}

\begin{abstract}
Dogs are not particularly known for complex physical cognitive abilities. However, a number of recent violation-ofexpectation studies have challenged this view. In the current eye-tracking study, we further investigated dogs' $(\mathrm{N}=15)$ reaction to physically implausible events, particularly in the context of support, occlusion, and launching events. In Experiment 1, the dogs watched a rolling ball moving over a gap in a surface either falling down or hovering over the gap. In Experiment 2, the dogs saw a ball rolling behind a narrow pole either disappearing behind it or re-appearing on the other side. In Experiment 3, the dogs observed launching events either with or without contact between the balls. The dogs' pupil dilation response and looking times suggest that they form implicit expectations about occlusion and launching events but not about gravity-related events at least in the context of animated objects on a screen.
\end{abstract}

Keywords: eye tracking; pupillometry; physical cognition; contact causality; object knowledge; expectancy violation; canine cognition

\section{Introduction}

Expectations about physical principles could help animals to navigate their environment more efficiently (e.g., Völter \& Call, 2017). To what extent dogs, one of the most popular model species in comparative cognitive research, are sensitive to physical principles such as support, solidity, connectivity, size constancy, or occlusion remains subject to ongoing debate (e.g., Müller, Riemer, Range, et al., 2014; Müller, Riemer, Virányi, et al., 2014; Osthaus et al., 2003; Tecwyn \& Buchsbaum, 2019).

Until recently, researchers suggested that dogs mostly rely on associative learning when solving problems in their physical environment (e.g., Collier-Baker et al., 2004; Fiset \& LeBlanc, 2007; Müller, Riemer, Range, et al., 2014). More recent evidence using the violation of expectation (VoE) paradigm challenged this conclusion. VoE paradigms capture the subjects' reaction to novel, potentially surprising events and can therefore be based on few or even a single trial per condition, which makes the findings harder to explain by associative learning accounts (with appropriate controls for perceptual novelty).

The VoE paradigm has been applied widely in the developmental and comparative literature to investigate expectations about the physical environment. For example, dogs reacted with increased smelling behavior after they had seen one food type being hidden inside a container but another food type reappearing from the container after a short delay (Bräuer \& Call, 2011). Moreover, dogs looked longer when objects changed their size (Müller et al., 2011; Pattison et al., 2013) or color behind a screen (Pattison et al., 2013) and even when a screen occluding a food reward rotated as though it had passed through the (hidden) reward (Pattison et al., 2010). These studies however relied on live demonstrations and manual scoring of looking times from the video, which can bias the results by introducing Clever-Hanseffects (i.e., inadvertently given cues by the experimenter for example; Pfungst, 1911; Schmidjell et al., 2012) and measurement errors. Eye tracking using standardized, videobased stimuli can serve to mitigate these issues.

Eye-tracking and pupillometry have been applied widely in cognitive research. In humans, eye movements are closely coupled to visual attention (e.g., Deubel \& Schneider, 1996; Hoffman \& Subramaniam, 1995). Dwell time (the sum of the fixation times in an area of interest) has been related to the processing difficulty and the predictability of a stimulus (Henderson, 2017). The psychosensory pupil dilation response has been linked to elevated arousal and mental effort (Mathôt, 2018). However, similar to other behavioral response variables (e.g. looking direction, reaction times) inferring specific cognitive processes from dwell times and pupil size changes is challenging and requires carefully controlled experiments given that a number of processes can lead to the same response (e.g., low-level perceptual aspects, novelty, familiarity, expectancy violations, etc.).

In the context of $\mathrm{VoE}$ studies with infants, pupil dilation has been suggested as a superior response variable (Jackson \& Sirois, 2009; Sirois \& Jackson, 2012). Particularly the temporal sensitivity of the phasic pupil dilation response but also its stability to test-order effects have been highlighted as advantage compared to looking time measures. In dogs only few eye-tracking studies so far looked at pupil size data: Somppi et al. (2017) found that dogs exhibited dilated pupils when presented with pictures of male human faces with an angry emotional expression compared to a happy expression. This effect was reversed when intranasal oxytocin was administered beforehand. This has been interpreted as evidence that oxytocin can modulate emotional arousal in dogs. Similarly, Karl et al. (2020) reported that dogs had larger pupils when they were presented with angry looking human faces than happy faces.

In the current eye-tracking study, we presented dogs with realistic 3D animations of moving balls. After a 
familiarization, the dogs saw one of two new test events that either violated certain physical principles or not. We ensured that the latter control events differed to the same or an even greater extent from the familiarization videos thereby controlling for novelty effects. In Experiment 1, we examined whether dogs would expect an unsupported object to fall down. In Experiment 2, we examined whether dogs would react with increased arousal when a rolling ball would disappear behind an object not wide enough to occlude it. In Experiment 3, we examined whether dogs would be sensitive to the principles of contact causality. Specifically, we investigated whether dogs would show increased arousal when they saw a contactless launching event.

\section{Methods}

\section{Subjects}

In Experiment 1, we tested 15 pet dogs ( 6 border collies, 5 mixed breeds, 2 Labrador Retrievers, 1 collie, and 1 Australian Shepherd; mean age: 2.8 years, range: 1-7 years; 9 females, 6 males). In Experiment 2 and 3, we tested the same dogs with the exception of one border collie that was unavailable $(\mathrm{N}=14$; mean age: 2.5 years, range: $1-6$ years; 8 females, 6 males).

The study was discussed and approved by the institutional ethics and animal welfare committee in accordance with GSP guidelines and national legislation (approval number: ETK066/03/2020).

\section{Stimuli}

In each experiment, we presented the dogs with a familiarization video and two different test videos. The videos showed 3D animations created in Blender 2.8 using Blender's rigid body physics simulation. The videos had a frame rate of $100 \mathrm{fps}$ and a duration of $3.5 \mathrm{~s}$ (Experiment 1 and 2) and $3.7 \mathrm{~s}$ (Experiment 3), respectively. However, in the eye-tracking experiments we extended presentation time of the last video frame for a total video duration of $4.5 \mathrm{~s}$ in the familiarization trials and $13.5 \mathrm{~s}$ in the test trials.

In Experiment 1, the familiarization video showed a yellow-black patterned ball rolling along an elevated light blue platform on a gray background. On the right side of the platform, we added a wall that served to stop the rolling ball at the end of the video. The video started with the ball partly visible on the left side of the screen and it ended with the ball bumping into wall. In the test videos, there was a gap in the elevated platform in the center of the screen. In the Hovering test condition (Figure 1B), the ball rolled along the platform and over the gap as before (i.e. on the same path and with the same kinematics as in the familiarization). In the Fallingdown test condition (Figure 1A), the ball fell into the gap and stopped when bumping into the right wall of the gap.

In Experiment 2, the familiarization video showed a bluesilver patterned ball rolling along a gray surface. A yellow rectangle occluded the right half of the screen. The ball started on the left side of the screen and rolled behind the opaque yellow wall. In the test videos, a slim yellow pole in the center of the screen replaced the yellow wall. In both test videos, the ball rolled as before from left to right. In the Reappear condition (Figure 2A), the ball rolled behind the pole, reappeared on the other side, rolled further to the right edge of the screen and moved out of view. In the Disappear condition (Figure 2B), the ball disappeared behind the pole.

In Experiment 3, the familiarization video showed a yellow-black patterned ball rolling along a gray surface from left to right. It got slower toward the right edge of the screen and stopped before it moved outside the field of view. In the test videos, there were two balls: again the yellow-black patterned ball starting on the left side of the screen (henceforth: launching ball) and a blue-white patterned ball (henceforth: target ball) closer to the center of the screen. The videos started with the launching ball rolling toward the inactive target ball. In the Contact condition (Figure 3A), the target ball was located closer to the launching ball than in the No-contact condition. In the Contact condition, the launching ball eventually hit the target ball, thereby, setting the latter into motion. As consequence of the collision event, the launching ball abruptly stopped moving while the target ball rolled toward the right edge of the screen. The target ball stopped moving before it moved out of view. In the Nocontact condition (Figure 3B), the launching and target balls moved with exactly the same kinematics as in the Contact condition. However, given that the two balls were further apart, the launching ball abruptly stopped moving and the target ball was set into motion without any contact between the two balls (or any other obvious cause).

\section{Apparatus}

We used the EyeLink1000 eye-tracking system (SR Research, Canada) to record the dogs' eye movements and pupil size at $1000 \mathrm{~Hz}$. We used an adjustable chin rest to facilitate the maintenance of a stable head position during stimulus presentation. We presented the stimuli on a 24 -inch LCD monitor (resolution: 1024 x 768; refresh rate: $100 \mathrm{~Hz}$ ) at a distance of $70 \mathrm{~cm}$ from the dogs' eyes. The video area subtended visual angles of 31.89 (horizontal) and 24.19 (vertical) degrees. The balls had a diameter of ca. $90 \mathrm{px}$ (Experiment 1), 105 px (Experiment 2), and $86 \mathrm{px}$ (Experiment 3) subtending a visual angle of 2.88, 3.36, and 2.75 degrees, respectively. We adjusted the height of the chin rest and the height and angle of the eye tracker for each subject.

\section{Design and Procedure}

The experiments used a within-subject design, in which we presented the subjects with two test conditions, one violating the physical principle under investigation and another one controlling for the novelty of the stimulus without violating this physical principle. The order of conditions was counterbalanced across subjects. We pseudo-randomly assigned the dogs to the order groups and counterbalanced the groups as much as possible with respect to age, sex, and 

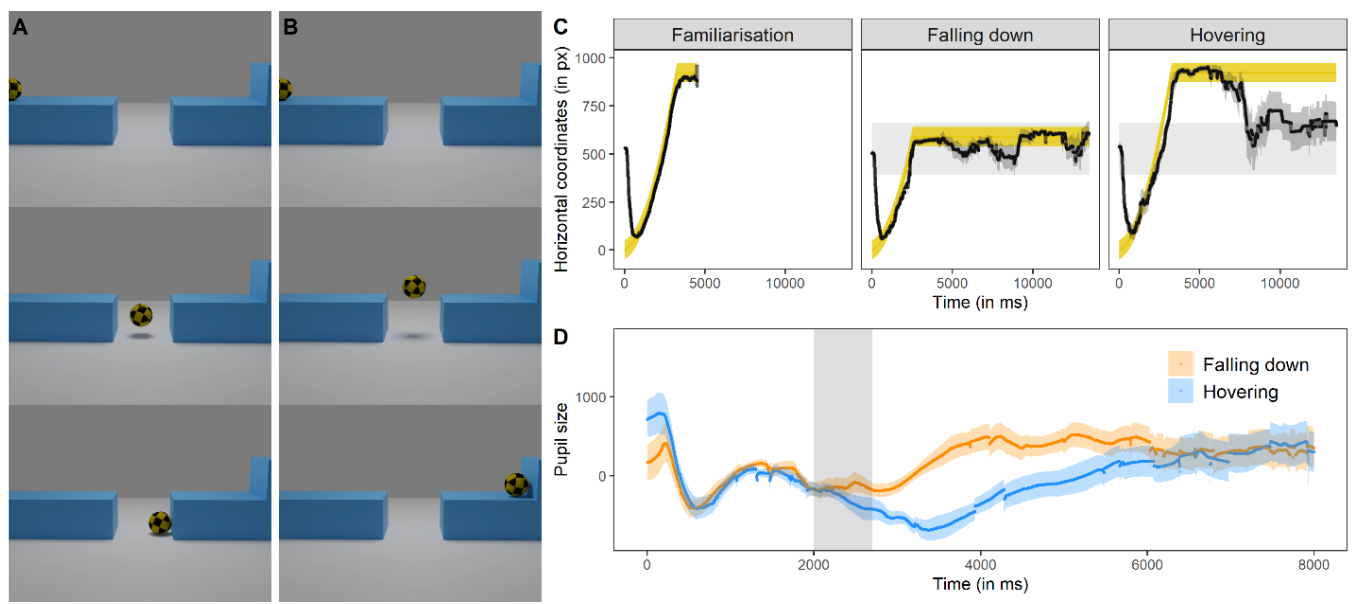

Figure 1 Screenshots of the Falling-down condition (A) and the Hovering condition (B). C. Time series plot showing the dogs' mean horizontal gaze coordinates ( \pm se, black line and the dark grey shaded area around it; in px) across the six familiarization trials and in the test trials. The shaded yellow area shows the left and right boundaries of the ball. The light grey shaded area indicates the position of the gap in the blue surface. D. Time series plot showing dogs' pupil size (in arbitrary units and baseline corrected) of the test videos in Experiment 1. The blue and orange lines show the mean pupil size ( \pm se, shaded area around the line) in the Hovering and Falling-down condition. The light grey shaded rectangle highlights the period in which the ball was hovering over the gap or falling into the gap.

breed. We conducted two sessions per dog. Each session consisted of three identical familiarization trials followed by one test trial. In total, we administered only one trial per test condition and six familiarization trials.

In each session, the dogs first completed a 5-point calibration with animated calibration targets $(24-64 \mathrm{px})$ subtending visual angles of $0.77-2.05$ degrees depending on the used target stimulus. Following the calibration, we presented a central fixation target (a white expanding circle; max diameter: 90 px; visual angle: 2.88 degrees). The video started once the dogs fixated the target for $50 \mathrm{msec}$.

\section{Analysis}

In order to analyze to what extent the horizontal coordinates of the moving ball predicted variance in dogs horizontal looking behavior, we calculated $\mathrm{r}^{2}$ values for the period between $500 \mathrm{~ms}$ after the onset of the video and the end of the movement of the ball (for the target ball in Experiment 3 we used the onset of the target ball motion as starting point). We determined the location of the balls by using the dynamic area of interest (AOI) recording function in EyeLink Data Viewer. We fitted a linear model for each subject with the dogs' horizontal gaze positions as the response variable and the $\mathrm{x}$-coordinates of the ball center as the predictor variable. We obtained the proportion of variance $\left(\mathrm{r}^{2}\right)$ explained by the horizontal ball coordinates from these models.

For the AoI analysis, we analyzed the interest period at the end of the video when the last frame of the video was shown for ca. $10 \mathrm{~s}$. We showed the last frame of the experiment for an extended period of time to allow for a dwell time analysis with the moving objects in their final position. In Experiment 1 and 3, we defined an AoI around the end positions of the (launching) ball (Exp1: w x h: 200 x 220 px; Exp3: w x h:
$240 \times 300$ px). In Experiment 2, the ball was not visible any more at the end of the video. Here, we analyzed an AoI around the pole ( $\mathrm{w} \times \mathrm{h}$ : $300 \mathrm{x} 460 \mathrm{px}$ ). We compared the dwell time in each AoI across conditions using two-tailed, paired-samples t-tests.

For the analysis of the pupil size data, we applied a subtractive baseline correction (Mathôt et al., 2018) by using the entire pre-event period (i.e. before the ball moved over the gap, moved behind the pole, or collided with target ball) of the video for the baseline correction. We aggregated the baseline-corrected pupil size data by calculating the dogs' mean pupil size in a 3 -s window starting $0.5 \mathrm{~s}$ after the onset of the critical test event (ball hovering in mid-air, occlusion, launch event), a response window within the range of the psychosensory pupil response reported in the human literature (Mathôt et al., 2018). We fitted a linear mixed model (LMM) to analyze the aggregated pupil size data. We included the predictor variables condition, order of condition, age (in months) as well as the mean visual angle of the gaze position measured from the screen center (in radians, arctangent of the distance of the dogs' gaze coordinates from the center of the screen divided by the distance between the dogs' right eye and the center of the screen).

The models were fitted using the $\mathrm{R}$ function lmer of the package lme4 (Bates et al., 2015). In addition to the fixed effects we included the random intercept subject ID as well as the random slope of the mean visual angle. We tested the effect of each fixed effect using likelihood ratio tests comparing the full model with reduced models lacking the respective fixed effect. We z-transformed all covariates to a mean of zero and a standard deviation of 1 . We found no obvious violations of the assumptions of normality and homogeneity of the residuals. Collinearity appeared to be 

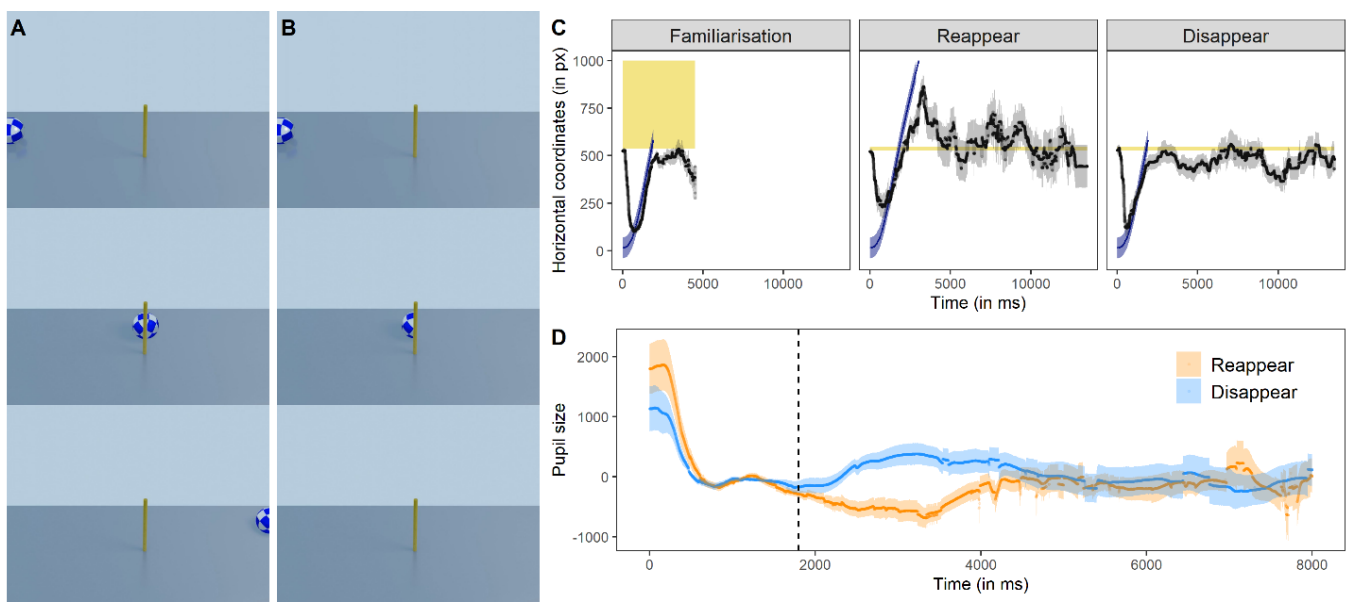

Figure 2 Screenshots of the Reappear condition (A) and the Disappear condition (B). C. Time series plot showing the dogs' mean horizontal gaze coordinates ( \pm se, black line and the dark grey shaded area around it; in px) across the familiarization trials and in the test trials. The shaded blue area around the line shows the left and right boundaries of the ball. The yellow shaded area indicates the area of the occluder / pole. D. Time series plot showing dogs' pupil size (in arbitrary units and baseline corrected). The blue and orange lines show the mean pupil size ( \pm se, shaded area around the line) in the Disappear and Reappear condition. The dashed vertical line indicates the time point when the center of the ball was behind the pole.

acceptable (maximum Variance Inflation Factor: Experiment 1: 3.17; Experiment 2: 1.29; Experiment 3: 1.06).

\section{Results}

\section{Experiment 1: Support}

We hypothesized that the dogs would react surprised to the Hovering test event (Figure 1B) if they were sensitive to the principle of support in this situation. In contrast, we hypothesized that the dogs would react more surprised to the Falling-down control condition (Figure 1A) if they merely formed expectations about the movement trajectory of the ball over the course of the familiarization. We therefore predicted that the dogs would exhibit pupil dilation response depending on their expectations about the behavior of the ball when it moved over the gap.

The dogs looked consistently on the screen while the video was playing (proportion of on-screen dwell time: Familiarization: median: 0.92, range: 0.19-0.96; Fallingdown: median: 0.92, range: 0.83-0.95; Hovering: median: 0.92 , range: $0.85-0.94)$. Their on-screen dwell time did not differ between the first and last familiarization trial $(\mathrm{t}(14)=0.47, \mathrm{p}=0.648)$ or between the two test conditions $(\mathrm{t}(14)=1.16, \mathrm{p}=0.267)$. They also closely followed the movement of the ball with their gaze in all conditions (Familiarization: median $\mathrm{r}^{2}$ : 0.88, range: 0.55-0.99; Fallingdown: median $\mathrm{r}^{2}$ : 0.94, range: 0.48-0.99; Hovering: median $r^{2}: 0.85$, range: $0.26-0.99$; Figure $1 C$ ). The AoI analysis of the interest period at the end of the video (with the ball in its end position) confirmed this: the dogs looked longer at the upper end position AoI in the Hovering condition compared to the Falling-down condition $(\mathrm{t}(14)=-4.69, \mathrm{p}<0.001)$. Conversely, dogs looked longer at the lower end position AoI in the Falling-down condition than in the Hovering condition $(\mathrm{t}(14)=5.13, \mathrm{p}<0.001)$. However, there was no difference in the dwell times for the respective ball end position AoIs between the two conditions $(\mathrm{t}(14)=0.59, \mathrm{p}=0.567)$.

LMM 01 revealed that dogs' pupils were significantly larger in the Falling-down condition compared to the Hovering condition $\left(\chi^{2}(1)=8.03, p=0.005\right.$; Figure 1D) in the interest period after the ball moved over the gap. The other predictor variables, order of condition $\left(\chi^{2}(1)=1.99, p=\right.$ $0.159)$, age $\left(\chi^{2}(1)=3.22, p=0.073\right)$, and mean visual angle $\left(\chi^{2}(1)=0.38, p=0.536\right)$, had no significant effect on the pupil size following the test event.

\section{Experiment 2: Occlusion}

We hypothesized that the dogs would show increased arousal in response to the Disappear test event (Figure 1B) compared to the Re-appear event (Figure 1A) if they were sensitive to the principles of occlusion (particularly with respect to the size of the occluder in relation to the size of the occluded object).

The dogs looked consistently on the screen while the video was playing (proportion of on-screen dwell time: Familiarization: median: 0.93, range: 0.24-0.98; Reappear: median: 0.92, range: 0.80-0.97; Disappear: median: 0.94, range: 0.89-0.98). Their on-screen dwell time did not differ between the first and last familiarization trial $(\mathrm{t}(13)=1.23$, $\mathrm{p}=0.239)$. But they looked longer on the screen in the Disappear condition than in the Reappear condition $(\mathrm{t}(13)=-$ 2.36, $\mathrm{p}=0.035$ ).

The dogs followed the movement of the ball in all conditions closely (Familiarization: median $\mathrm{r}^{2}$ : 0.71, range: 0.12-0.86; Reappear: median $\mathrm{r}^{2}$ : 0.69, range: 0.004-0.98; Disappear: median $\mathrm{r}^{2}$ : 0.71, range: 0.06-0.91). The AoI analysis revealed that the dogs looked significantly longer at the pole AoI in the interest period at the end of the video 

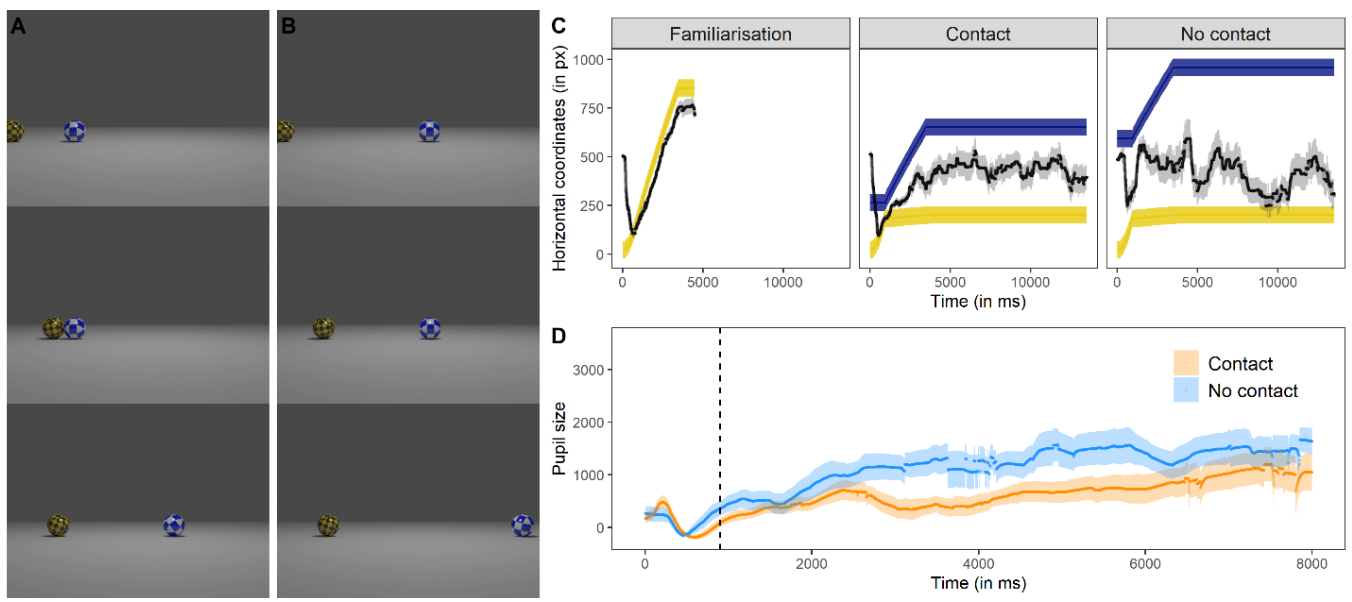

Figure 3 Screenshots of the Contact condition (A) and No-contact condition (B). C. Time series plot showing the dogs' mean horizontal gaze coordinates ( \pm se, black line and the dark grey shaded area around it; in px) across the familiarization trials and in the test trials. The shaded yellow area shows the left and right boundaries of the launching ball. The shaded blue area shows the left and right boundaries of the target ball. D. Time series plot showing dogs' pupil size (in arbitrary units and baseline corrected. The orange and blue lines show the mean pupil size ( \pm se, shaded area around the line $)$ in the Contact and No-contact condition. The dashed vertical line indicates the time point when the target ball started moving.

(when the ball was no longer visible) in the Disappearing condition than in the Reappearing condition $(\mathrm{t}(13)=2.37$, $\mathrm{p}=0.034)$.

LMM 02 revealed that dogs' pupils were significantly larger in the Disappearing condition compared to the Reappearing control condition $\left(\chi^{2}(1)=7.20, p=0.007\right.$; Figure 2D). The other predictor variables, order of condition $\left(\chi^{2}(1)=0.90, p=0.342\right)$, age $\left(\chi^{2}(1)=0.61, p=0.436\right)$, and mean visual angle $\left(\chi^{2}(1)=1.29, p=0.255\right)$, had no significant effect on the pupil size following the test event.

\section{Experiment 3: Contact causality}

We hypothesized that the dogs would find the No-contact condition more surprising than the Contact condition if they were sensitive to the principles of contact causality. Accordingly, we predicted that the dogs would show pupil dilation in response to the No-contact test event compared to the Contact condition.

The dogs looked consistently on the screen while the video was playing (proportion of on-screen dwell time: Familiarization: median: 0.92, range: 0.75-0.98; Contact: median: 0.93 , range: $0.88-0.97$; No-contact: median: 0.93, range: $0.85-0.99)$. Their on-screen dwell time did not differ between the first and last familiarization trial $(\mathrm{t}(13)=0.66$, $\mathrm{p}=0.522)$ or between the two test conditions $(\mathrm{t}(13)=-0.79$, $\mathrm{p}=0.443)$. In the familiarization, dogs followed the rolling ball closely (median $\mathrm{r}^{2}: 0.87$, range: $0.18-0.98$; Figure $3 \mathrm{C}$ ). In the test trials, the dogs for the most part also followed the movement of the launch ball in both conditions (Contact: median $r^{2}$ : 0.73, range: 0.12-0.93; No-contact: median $r^{2}$ : 0.66 , range: $0-0.91$ ). When the target ball started moving some dogs also looked back and forth between the balls, which led to smaller $\mathrm{r}^{2}$ values for the target ball (Contact: median $\mathrm{r}^{2}: 0.21$, range: 0-0.96; No-contact: median $\mathrm{r}^{2}: 0.54$, range:0-0.84). The dogs looked significantly longer at the launch ball end position AoI in the end interest period (when balls did not move anymore) in the No-contact condition compared to the Contact control condition $(t(13)=-2.66, p=$ $0.020)$.

LMM 03 revealed that dogs' pupils were significantly larger in the No-contact condition compared to the Contact control condition $\left(\chi^{2}(1)=5.14, p=0.023\right.$; Figure 3D). The other predictor variables, order of condition $\left(\chi^{2}(1)=0.08, p=\right.$ $0.0 .777)$, age $\left(\chi^{2}(1)=2.25, p=0.134\right)$, and mean visual angle $\left(\chi^{2}(1)=1.47, p=0.225\right)$, had no significant effect on the pupil size following the test event.

\section{Discussion}

The dogs followed the animated balls closely with their gaze throughout the three experiments. Our results indicate that dogs' pupils dilated in response to changing movement trajectories, the sudden disappearance, and the balls' sudden motion onset and offset without any direct contact between them. The dwell time data supported this pattern with longer dwell times to the pole behind which the ball disappeared (Experiment 2) and to the launching ball that stopped moving without any contact with the target ball (Experiment 3 ). In contrast, we found no evidence that the dogs had gravityrelated expectations about unsupported ball falling down (Experiment 1).

In line with our results of Experiment 1, previous research studies did not provide evidence for a clear gravity bias in dogs, i.e. the tendency to look for a dropped object directly underneath the place where they have seen it last (Osthaus et al., 2003; Tecwyn \& Buchsbaum, 2019). Nevertheless, it would be interesting to explore in future eye-tracking experiments whether dogs would show a pupil dilation response if the gravity violation was shown for longer period 
of time at the end of the demonstration (like in previous comparative and developmental studies; e.g., Baillargeon et al., 1992; Bird \& Emery, 2010; Cacchione \& Krist, 2004; Murai et al., 2011). Additionally, the sudden change in direction in the Falling-down control condition might have led to a dishabituation. However, if the dogs had the expectation of unsupported objects to fall down they should have expected this sudden downward movement when the ball moved over the gap.

Previous comparative research using violations of support relations found that chimpanzees (Cacchione \& Krist, 2004; Murai et al., 2011) and Japanese macaques (Murai et al., 2011) showed longer looking times when an object was hovering in the air without any contact to a surface than when it was in contact with a surface. However, the primates did not distinguish between vertical contact (an unsupported object placed against a vertical surface) and an object on top of a horizontal surface (indicating support). Rooks, in contrast, not only looked longer at pictures of hovering objects without any contact with a surface but also when the object only made contact with a surface without being supported by the surface (Bird \& Emery, 2010).

Dogs' pupil dilation reaction to the Disappear condition in Experiment 2 provides evidence that they were surprised by the sudden disappearance of the ball behind the pole. We observed this pupil dilation response even though the ball in the familiarization trials also disappeared at the same location (but in contrast to the test events behind a wider screen). This finding fits to previous work using occlusion events. Dogs reacted with increased smelling behavior when a food item hidden inside a container was "magically" replaced by different food item while out of sight (Bräuer \& Call, 2011). In other studies, dogs looked longer when the occluded object changed its size or color while being occluded (Müller et al., 2011; Pattison et al., 2013). In another VoE study, dogs who had witnessed a toy or reward placed behind a screen looked longer when the screen rotated as if no reward was present behind it (Pattison et al., 2010). Together these studies suggest that dogs remember some properties of the hidden item and expect that the hidden object will constrain the movements of the overlying screen. The current study indicates that dogs might also consider the size of the occluding object in relation the occluded object (similar to previous findings with human infants, e.g. Luo \& Baillargeon, 2005).

Experiment 3 is to our knowledge the first investigation of dogs' expectations about contact causality. Previous research has shown that human infants that were habituated to normal launching events dishabituated when they were presented with launching without contact events comparable to the Nocontact condition of the current study (Leslie, 1984). Future studies should examine whether also temporal lags between the contact event and the onset of the target motion can induce a pupil dilation response in dogs.

Dwell time and the psychosensory pupil response are only indirectly linked to cognitive abilities or mental states. And they are unspecific indicators, i.e., many factors can lead to a similar dwell time or pupil size response (Mathôt, 2018). Thus, other factors such as low-level perceptual aspects might provide alternative explanations for the current findings. For example, a sudden change in the movement direction might explain the pupil dilation in response to the falling ball in Experiment 1. A sudden stop of the moving stimulus or a decrease in optical flow might explain the pupil dilation in response to the disappearing ball in Experiment 2 . Follow-up studies will be needed to address such alternative explanations by further matching experimental and control conditions with respect to these perceptual aspects. The Hovering (Experiment 1) and Reappear (Experiment 2) conditions could be changed such that the ball stops moving at the same horizontal position and time point as in the Falling-down (Experiment 1) and Disappear (Experiment 2) conditions. For instance, in the case of occlusion events, the ball could be stopped right after passing the occluder instead of continuing to move.

Furthermore, positive findings in looking time or pupillometric studies do not necessarily translate into successful performance in choice or search tasks, a lesson learnt from both developmental and comparative research (e.g., Hood et al., 2000; Santos \& Hauser, 2002). Therefore, our findings do not necessarily indicate that dogs would also form their decisions based on an understanding of occlusion events or contact causality. Indeed previous research with search and choice tasks provide reasons to doubt that dogs would adjust their search behavior accordingly (CollierBaker et al., 2004; Fiset \& LeBlanc, 2007; Müller, Riemer, Range, et al., 2014).

In summary, our findings are consistent with the notion that dogs form implicit expectations based on certain physical principles and show surprise if these are violated. This appears to be the case for occlusion events and contact causality. Our results also show limitations in that the dogs' pupil size reaction did provide evidence for gravity-related expectations, i.e. that they would expect unsupported objects to fall down. However, follow-up studies will be needed to rule out low-level perceptual cues as alternative explanations. At a methodological level, our study highlights the potential of pupillometry for the investigation of expectancy violations in dogs.

\section{Acknowledgements}

We thank Laura Laussegger and Sabrina Karl for their help with training and the data collection, Karin Bayer for administrative support, Sam Hutton (SR Research), Wolfgang Berger, and Peter Füreder for technical support, and Roger Mundry for his advice concerning the statistical analyses. Furthermore, we are grateful to the dog caregivers for participating with their dogs in this study.

This project was supported by the Austrian Science Fund (FWF) through project W1262-B29 and by the Vienna Science and Technology Fund (WWTF), the City of Vienna and ithuba Capital AG through project CS18-012. 


\section{References}

Baillargeon, R., Needham, A., \& Devos, J. (1992). The development of young infants' intuitions about support. Early Development and Parenting, 1(2), 69-78.

Bates, D., Mächler, M., Bolker, B., \& Walker, S. (2015). Fitting Linear Mixed-Effects Models Using Ime4. Journal of Statistical Software, 67(1), 1-48.

Bird, C. D., \& Emery, N. J. (2010). Rooks perceive support relations similar to six-month-old babies. Proceedings of the Royal Society B: Biological Sciences, 277(1678), 147151.

Bräuer, J., \& Call, J. (2011). The magic cup: Great apes and domestic dogs (Canis familiaris) individuate objects according to their properties. Journal of Comparative Psychology, 125(3), 353-361.

Cacchione, T., \& Krist, H. (2004). Recognizing Impossible Object Relations: Intuitions About Support in Chimpanzees (Pan troglodytes). Journal of Comparative Psychology, 118(2), 140-148.

Collier-Baker, E., Davis, J. M., \& Suddendorf, T. (2004). Do dogs (Canis familiaris) understand invisible displacement? Journal of Comparative Psychology, 118(4), 421-433.

Deubel, H., \& Schneider, W. X. (1996). Saccade target selection and object recognition: Evidence for a common attentional mechanism. Vision Research, 36(12), 18271837.

Fiset, S., \& LeBlanc, V. (2007). Invisible displacement understanding in domestic dogs (Canis familiaris): The role of visual cues in search behavior. Animal Cognition, 10, 211-224.

Henderson, J. M. (2017). Gaze Control as Prediction. Trends in Cognitive Sciences, 21(1), 15-23.

Hoffman, J. E., \& Subramaniam, B. (1995). The role of visual attention in saccadic eye movements. Perception \& Psychophysics, 57(6), 787-795.

Hood, B., Carey, S., \& Prasada, S. (2000). Predicting the Outcomes of Physical Events: Two-Year-Olds Fail to Reveal Knowledge of Solidity and Support. Child Development, 71(6), 1540-1554.

Jackson, I., \& Sirois, S. (2009). Infant cognition: Going full factorial with pupil dilation. Developmental Science, 12(4), 670-679.

Karl, S., Boch, M., Zamansky, A., van der Linden, D., Wagner, I. C., Völter, C. J., Lamm, C., \& Huber, L. (2020). Exploring the dog-human relationship by combining fMRI, eye-tracking and behavioural measures. Scientific Reports, 10(1), 22273.

Leslie, A. M. (1984). Spatiotemporal Continuity and the Perception of Causality in Infants. Perception, 13(3), 287305.

Luo, Y., \& Baillargeon, R. (2005). When the ordinary seems unexpected: Evidence for incremental physical knowledge in young infants. Cognition, 95(3), 297-328.

Mathôt, S. (2018). Pupillometry: Psychology, Physiology, and Function. Journal of Cognition, 1(1), 16.

Mathôt, S., Fabius, J., Van Heusden, E., \& Van der Stigchel, S. (2018). Safe and sensible preprocessing and baseline correction of pupil-size data. Behavior Research Methods, 50(1), 94-106.

Müller, C. A., Mayer, C., Dörrenberg, S., Huber, L., \& Range, F. (2011). Female but not male dogs respond to a size constancy violation. Biology Letters, 7, 689-691.

Müller, C. A., Riemer, S., Range, F., \& Huber, L. (2014). Dogs' use of the solidity principle: Revisited. Animal Cognition, 17(3), 821-825.

Müller, C. A., Riemer, S., Virányi, Z., Huber, L., \& Range, F. (2014). Dogs learn to solve the support problem based on perceptual cues. Animal Cognition, 17, 1071-1080.

Murai, C., Tanaka, M., \& Sakagami, M. (2011). Physical intuitions about support relations in monkeys (Macaca fuscata) and apes (Pan troglodytes). Journal of Comparative Psychology, 125(2), 216-226.

Osthaus, B., Slater, A. M., \& Lea, S. E. G. (2003). Can dogs defy gravity? A comparison with the human infant and a non-human primate. Developmental Science, 6(5), 489497.

Pattison, K. F., Laude, J. R., \& Zentall, T. R. (2013). The case of the magic bones: Dogs' memory of the physical properties of objects. Learning and Motivation, 44(4), 252-257.

Pattison, K. F., Miller, H. C., Rayburn-Reeves, R., \& Zentall, T. (2010). The case of the disappearing bone: Dogs' understanding of the physical properties of objects. Behavioural Processes, 85(3), 278-282.

Pfungst, O. (1911). Clever Hans (the horse of Mr. Von Osten.) A contribution to experimental animal and human psychology. Holt, Rinehart and Winston.

Santos, L. R., \& Hauser, M. D. (2002). A non-human primate's understanding of solidity: Dissociations between seeing and acting. Developmental Science, 5(2), F1-F7.

Schmidjell, T., Range, F., Huber, L., \& Virányi, Z. (2012). Do Owners Have a Clever Hans Effect on Dogs? Results of a Pointing Study. Frontiers in Psychology, 3(558).

Sirois, S., \& Jackson, I. R. (2012). Pupil dilation and object permanence in infants. Infancy, 17(1), 61-78.

Somppi, S., Törnqvist, H., Topál, J., Koskela, A., Hänninen, L., Krause, C. M., \& Vainio, O. (2017). Nasal oxytocin treatment biases dogs' visual attention and emotional response toward positive human facial expressions. Frontiers in Psychology, 8, 1854.

Tecwyn, E. C., \& Buchsbaum, D. (2019). What factors really influence domestic dogs' (Canis familiaris) search for an item dropped down a diagonal tube? The tubes task revisited. Journal of Comparative Psychology, 133(1), 4 19.

Völter, C. J., \& Call, J. (2017). Causal and inferential reasoning in animals. In J. Call, G. M. Burghardt, I. M. Pepperberg, C. T. Snowdon, \& T. Zentall (Eds.), APA handbook of comparative psychology: Perception, learning, and cognition. American Psychological Association. 